SCIENTIFIC LETTER

\title{
Evidence for association between hepatitis $C$ virus seropositivity and coronary artery disease
}

\author{
C Vassalle, S Masini, F Bianchi, G C Zucchelli
}

$\mathrm{T}$ he inflammation hypothesis of atherosclerosis postulates that the crucial events involved in the initiation and progression of the lesion are represented mainly by inflammatory and fibroproliferative processes triggered by cytokines and growth factors. ${ }^{1}$ However, one of the most interesting developments in recent years has been the idea that infective agents may induce a pro-inflammatory effect and have a crucial role in atherothrombosis. ${ }^{1}$ In addition, at the beginning of the 1970s, the monoclonal hypothesis was first proposed, suggesting a potential role for viral infection in the atherosclerotic process. ${ }^{2}$ In particular, this theory proposed that a mutation or a viral agent may represent events able to transform a single smooth muscle cell into the progenitor of a proliferative clone, introducing the concept that the plaque may be considered a monoclonal benign neoplasm. Since then, many additional results have shown an association between infectious agents and the atherosclerotic process, although other studies have produced contrary results. ${ }^{1}$ Thus, the role of infection in the pathogenesis of this disease remains controversial. In particular, very few data on the relation between hepatitis C virus (HCV) infection and atherosclerosis are available. However, very recent results indicate that seropositivity for HCV shows a positive association with carotid artery plaque and carotid intima-media thickening, independent from other risk factors for atherosclerosis. ${ }^{3}$ In the present study, we evaluated whether seropositivity for HCV is associated with the occurrence of coronary artery disease (CAD).

\section{METHODS}

The enrolment of patients was conducted according to previously described criteria. ${ }^{4}$ Our study population included 491 patients ( 92 females, 399 males), with a mean (SEM) age of $66(0.5)$ years and with angiographic documentation of CAD ( $>50 \%$ stenosis). A control group of 195 patients (8 females, 115 males) with a mean age of 61 (11.6) years was also recruited; these patients were hospitalised at the same institution for reasons other than suspected CAD (that is, valvar heart disease, cardiomyopathy, and hypertensive heart disease) and had angiographically documented normal coronary arteries. At the time of blood sampling, all subjects gave a complete history which included cardiovascular risk factors such as smoking habits, hypertension, diabetes and dyslipidaemia. Each patient was found to be negative for hepatitis B, as evaluated by anti-hepatitis B surface antigen positivity. Patient exclusion criteria were severe liver damage and cirrhosis, acute or chronic inflammatory disease, immunological disease, and history or presence of neoplastic disease. In addition, patients with a stenosis $<50 \%$, a stenosis on a minor vessel, or with atypical chest pain were excluded from the study.

Venous blood samples were collected under standardised conditions after an overnight fast and centrifuged within 15 minutes $(3000 \mathrm{~g}$ for 10 minutes). Serum samples were immediately analysed for measurements of antibodies against HCV (AXSYM System, Abbott Laboratories, Illinois, USA). Intra-run and inter-run coefficient of variation (\%CV) results were always $<8 \%$. Specificity, calculated in 4383 blood donors, was found to be $98.84 \%$. Data were expressed as the mean (SEM). Statistical analyses were performed using unpaired $t$ tests, $\chi^{2}$ tests, and univariate and multivariate logistic regression (Statview statistical package, version 5.0.1, SAS Institute). A probability value of $\mathrm{p} \leqslant 0.05$ was considered significant.

\section{RESULTS}

The percentage of HCV seropositivity was $2 \%$ in control subjects and $6.3 \%$ in the CAD group $\left(\chi^{2}=5.3, \mathrm{p}<0.05\right)$, increasing with the number of vessels affected $(4.5 \%$ for one vessel disease, $6.6 \%$ for two vessel disease, and $8.4 \%$ for three vessel disease, $\mathrm{p}<0.05)$. Results of the univariate logistic regression analysis showed that in addition to other conventional atherogenic risk factors (age, sex, smoking habit, hypertension, diabetes, and dyslipidaemia), HCV seropositivity was found to be associated with the presence of CAD with an odds ratio of 3.2 (95\% confidence interval (CI) 1.1 to 9.2, $\mathrm{p}<0.05$ ) (table 1). After adjustment for other confounding risk factors, the multivariate logistic regression analysis

Abbreviations: $C A D$, coronary artery disease; $H C V$, hepatitis $C$ virus

Table 1 Predictors for coronary artery disease

\begin{tabular}{lll}
\hline & \multicolumn{2}{l}{ Odds ratio $(95 \% \mathrm{Cl})$} \\
\cline { 2 - 3 } & Unadjusted & Adjusted* \\
\hline Age & $1.1(1.03$ to 1.1$), \mathrm{p}<0.001$ & $1.1(1.04$ to 1.1$), \mathrm{p}<0.001$ \\
HCV seropositivity & $3.2(1.1$ to 9.2$), \mathrm{p}<0.05$ & $4.2(1.4$ to 13$), \mathrm{p}<0.05$ \\
Sex & $3.0(2.1$ to 4.3$), \mathrm{p}<0.001$ & $4.1(2.6$ to 6.3$), \mathrm{p}<0.001$ \\
Smoking habit & $1.6(1.1$ to 2.2$), \mathrm{p}<0.01$ & $1.2(0.9$ to 1.8$), \mathrm{p}=0.3$ \\
Hypertension & $1.3(1.0$ to 1.8$), \mathrm{p}=0.08$ & $1.3(0.9$ to 1.9$), \mathrm{p}=0.2$ \\
Diabetes & $2.4(1.4$ to 3.8$), \mathrm{p}<0.001$ & $2.8(1.6$ to 4.7$), \mathrm{p}<0.001$ \\
Dyslipidaemia & $3.0(2.1$ to 4.3$), \mathrm{p}<0.001$ & $4.0(2.7$ to 5.9$), \mathrm{p}<0.001$ \\
\hline
\end{tabular}

*Odds ratio derived from logistic regression analysis including all the variables listed in the table. 
showed that HCV seropositivity still represented an independent predictor for CAD with an odds ratio of 4.2 (95\% CI 1.4 to $13.0, \mathrm{p}<0.05$ ) (table 1).

\section{DISCUSSION}

Several studies have suggested that some infectious agents may cause cellular and molecular changes that contribute to the pathogenesis of atherosclerosis. ${ }^{1}$ The data obtained indicate the identification of viral genomes in the atherosclerotic plaques and also pro-atherogenic effects of viral infection in cells relevant to atherogenesis (smooth muscle cells, monocyte macrophages, $\mathrm{T}$ cells, endothelial cells). Experimental models have also shown promotion and acceleration of atherosclerosis by infectious agents. ${ }^{1}$ Recent results indicate that infection by several different pathogens can confer high risk in both early and advanced atherogenesis (the first considered as the development of new plaques and the latter defined by the development/progression ratio of carotid stenosis). ${ }^{5}$ However, very little data about the possible relation between HCV infection and its associated putative pathogenic processes and atherosclerosis are available. Very recent data have indicated that seropositivity for HCV may have a role in the pathogenesis of carotid atherosclerosis. ${ }^{3}$ Our findings suggest that HCV seropositivity might be considered, in the clinical setting, as one of the risk factors affecting the onset and development of CAD. Further studies are needed at this point to verify the potential additive effect of HCV infection with respect to the presence of other pathogens. This study might be relevant for adding new predictive and prognostic factors to the CAD multifactorial entity.

\section{Authors' affiliations \\ C Vassalle, S Masini, F Bianchi, G C Zucchelli, Institute of Clinical Physiology, CNR, Pisa, Italy}

Correspondence to: Dr Cristina Vassalle, Istituto di Fisiologia ClinicaArea di ricerca CNR, Via Moruzzi 1, I-56100, Pisa, Italy; cristina.vassalle@ifc.cnr.it

Accepted 25 September 2003

\section{REFERENCES}

1 Shah PK. Link between infection and atherosclerosis: who are the culprits: viruses, bacteria, both, or neither? Circulation 2001;103:5-6.

2 Benditt EP, Benditt JM. Evidence for a monoclonal origin of human atherosclerotic plaques. Proc Natl Acad Sci U S A 1973;70:1753-6.

3 Ishizaka N, Ishizaka $Y$, Takahashi $E$, et al. Association between hepatitis C virus seropositivity, carotid-artery plaque, and intima-media thickening Lancet 2002;359:133-5.

4 Vassalle C, Botto N, Andreassi MG et al. Evidence for enhanced 8isoprostane plasma levels, as index of oxidative stress in vivo, in patients with coronary artery disease. Coron Artery Dis 2003;14:213-8.

5 Kiechl S, Egger G, Mayr M, et al. Chronic infections and the risk of carotid atherosclerosis: prospective results from a large population study. Circulation $2001 ; 103: 1064-70$.

\section{IMAGES IN CARDIOLOGY}

doi: $10.1136 /$ hrt.2002/006874

\section{Sirolimus eluting stent aborted recurrent distal left main in-stent restenosis involving bifurcation} eft main coronary artery stenosis is associated with dismal prognosis -when untreated. Coronary artery bypass grafting (CABG) is the standard treatment. Although stent implantation is increasingly performed for de novo left main lesion, CABG is inevitable if in-stent restenosis (ISR) occurs. Recently, use of sirolimus eluting stents has proved effective in reducing restenosis in simple de novo lesions. We present a patient who underwent sirolimus eluting stent implantation for significant left main ISR involving the bifurcation.

The patient was a 60 year old woman who presented with exertional angina caused by isolated ostial left anterior descending artery (LAD) stenosis. Direct bare stent implantation was initially performed, but was followed by occurrence of diffuse ISR six months later. She was then treated by additional bare stent implantation (stent-in-stent), with the second stent across the left main coronary artery. Six months repeat coronary angiography showed recurrence of ISR with involvement of the distal left main coronary artery and ostial left circumflex (LCX) artery (below, left panel).

After sequential balloon predilation, two sirolimus eluting stents (Cypher, Cordis) were deployed in the left main $\operatorname{LAD}(3.0 \times 18 \mathrm{~mm})($ parent vessel $) / \mathrm{LCX}$ $(3.0 \times 8 \mathrm{~mm})$ (side branch) bifurcation lesion by $\mathrm{T}$ stent technique. The stents were post-dilated with kissing balloon angioplasty. Final angiography showed good angiographic results (below, middle panel). The patient was prescribed aspirin and clopidogrel for six months. Six month follow up angiography (below, right panel) and intravascular ultrasound (upper right panel) showed no evidence of restenosis. Therefore, our case illustrates that the use of sirolimus eluting stents can be effective in the most complex lesion subset-distal left main ISR involving bifurcation.

\section{$\mathrm{C} \mathrm{H}$ Lee serruys@card.azr.nl}

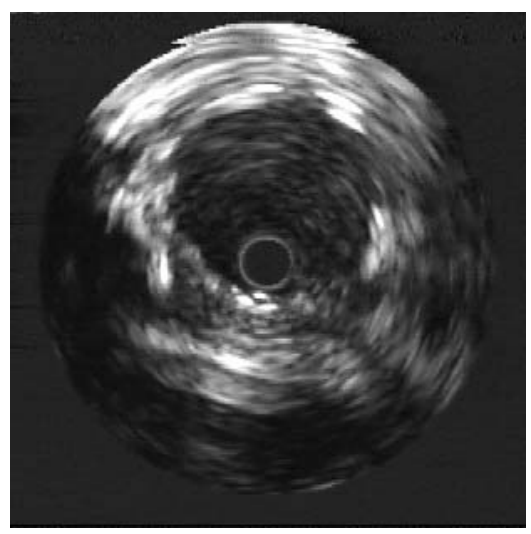

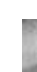

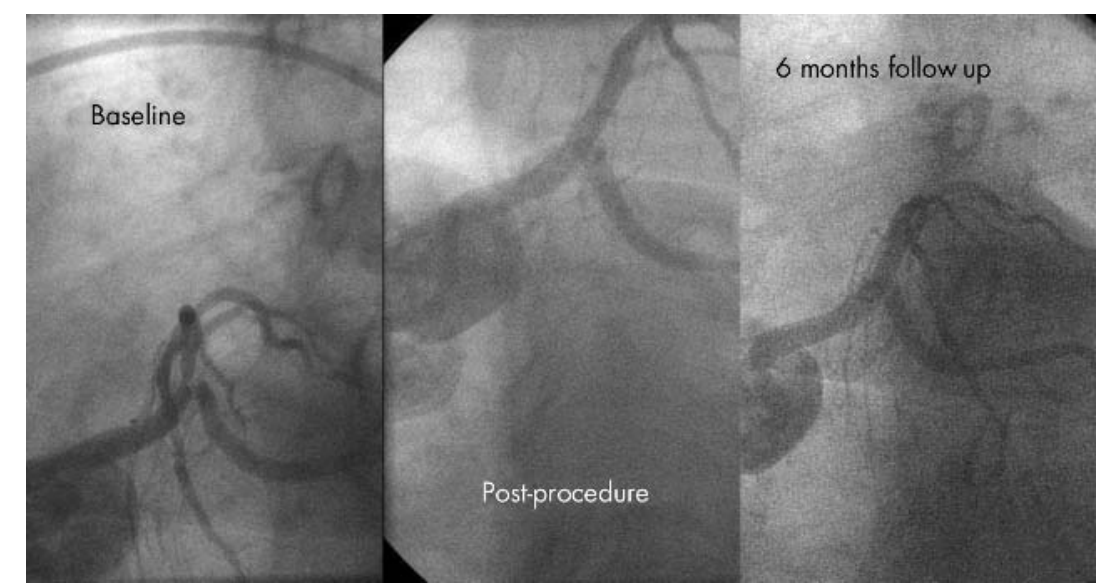

